\title{
Three solutions to a $p(x)$-Laplacian problem in weighted-variable-exponent Sobolev space
}

\author{
Wen-Wu Pan, Ghasem Alizadeh Afrouzi and Lin Li
}

\begin{abstract}
In this paper, we verify that a general $p(x)$-Laplacian Neumann problem has at least three weak solutions, which generalizes the corresponding result of the reference [R. A. Mashiyev, Three Solutions to a Neumann Problem for Elliptic Equations with Variable Exponent, Arab. J. Sci. Eng. 36 (2011) 1559-1567].
\end{abstract}

\section{Introduction}

Recently, elliptic equations with variable exponents have been extensively investigated and have received much attention. They have been the subject of recent developments in nonlinear elasticity theory and electrorheological fluids dynamics [16]. In that context, let us mention that there appeared a series of papers on problems which lead to spaces with variable exponent, we refer the reader to Fan et al. [8,9], Ruzicka [16] and the references therein.

Let us point out that when $p(x)=p=$ constant, there is a large literature which deal with problems involving the $p$-Laplacian with Dirichlet boundary conditions both in bounded or unbounded domains, which we do not need to cite here since the reader may easily find such papers.

Note that many papers deal with problems related to the $p$-Laplacian with Neumann conditions in the scalar case. We can cite, among others, the articles $[1,4]$ and refer to the references therein for details. The case of $p(x)$-Laplacian

Key Words: $p(x)$-Laplacian problems, Neumann problems, Ricceri's variational principle 2010 Mathematics Subject Classification: Primary 34B15; Secondary 35A15, 35G99.

Received: January 2012

Accepted: May 2013 
with Neumann conditions has been studied by Dai [6], Mihailescu [13] and Liu [11].

In this paper, we will consider the Neumann problems involving the $p(x)$ Laplacian operator

$$
\begin{cases}-\operatorname{div}\left(|\nabla u|^{p(x)-2} \nabla u\right)+a(x)|u|^{p(x)-2} u=\lambda f(x, u)+\mu g(x, u), & \text { in } \Omega, \\ \frac{\partial u}{\partial \nu}=0, & \text { on } \partial \Omega,\end{cases}
$$

where $\Omega \subset \mathbb{R}^{N}(N \geq 3)$ is a bounded domain with smooth boundary, $\lambda, \mu>0$ are real numbers, $p(x)$ is a continuous function on $\bar{\Omega}$ with $\inf _{x \in \bar{\Omega}} p(x)>N$ and $a \in L^{\infty}(\Omega)$ with $\operatorname{essinf}_{x \in \Omega} a(x)=a_{0}>0$. We denote by $\nu$ the outward unit normal to $\partial \Omega$. The main interest in studying such problems arises from the presence of the $p(x)$-Laplacian operator $\operatorname{div}\left(|\nabla u|^{p(x)-2} \nabla u\right)$, which is a generalization of the classical $p$-Laplacian operator $\operatorname{div}\left(|\nabla u|^{p-2} \nabla u\right)$ obtained in the case when $p$ is a positive constant.

When $\mu=0$, in [12], R. A. Mashiyev studied the particular case

$$
f(t)=b|t|^{q-2} t-d|t|^{s-2} t
$$

where $b$ and $d$ are positive constants, $2<s<q<\inf _{x \in \bar{\Omega}} p(x)$ and $N<$ $\inf _{x \in \bar{\Omega}} p(x) ;$ and

$$
f(x, t)=|t|^{q(x)-2} t-|t|^{s(x)-2} t
$$

where

$$
2<\inf _{x \in \bar{\Omega}} s(x) \leq \sup _{x \in \bar{\Omega}} s(x)<\inf _{x \in \bar{\Omega}} q(x) \leq \sup _{x \in \bar{\Omega}} q(x)<\inf _{x \in \bar{\Omega}} p(x)
$$

and $N<\inf _{x \in \bar{\Omega}} p(x)$ for all $x \in \bar{\Omega}$. He established the existence of at least three weak solutions by using the Ricceri's variational principle.

In this paper, we assume $f(x, u)$ and $g(x, u)$ satisfies the following general conditions:

(f1) $f, g: \Omega \times \mathbb{R} \rightarrow \mathbb{R}$ are Carathéodory functions and satisfies

$$
\begin{array}{rlrl}
|f(x, t)| & \leq c_{1}+c_{2}|t|^{\alpha(x)-1}, & & \forall(x, t) \in \Omega \times \mathbb{R}, \\
|g(x, t)| \leq c_{1}^{\prime}+c_{2}^{\prime}|t|^{\beta(x)-1}, & \forall(x, t) \in \Omega \times \mathbb{R},
\end{array}
$$

where $\alpha(x), \beta(x) \in C(\bar{\Omega}), \alpha(x), \beta(x)>1$ and $1<\alpha^{+}=\max _{x \in \bar{\Omega}} \alpha(x)<$ $p^{-}=\min _{x \in \bar{\Omega}} p(x), 1<\beta^{+}=\max _{x \in \bar{\Omega}} \beta(x)<p^{-}=\min _{x \in \bar{\Omega}} p(x)$ and $c_{1}$, $c_{2}, c_{1}^{\prime}, c_{2}^{\prime}$ are positive constants. 
(f2) There exist a constant $t_{0}$ and following conditions satisfies

$$
\begin{array}{ll}
f(x, t)<0 & \text { when }|t| \in\left(0, t_{0}\right) \\
f(x, t)>M>0 & \text { when }|t| \in\left(t_{0},+\infty\right),
\end{array}
$$

where $M$ is a positive constant.

Following along the same lines as in [12], we will prove that there also exist three weak solutions for such a general problem for $\lambda$ sufficiently large and requiring $\mu$ small enough.

\section{Preliminary results and lemma}

In this part, we introduce some theories of Lebesgue-Sobolev space with variable exponent. The detailed description can be found in $[10,17,8,9]$. Denote by $S(\Omega)$ the set of all measurable real functions on $\Omega$. Set

$$
C_{+}(\bar{\Omega})=\{p: p \in C(\bar{\Omega}), p(x)>1, \forall x \in \bar{\Omega}\} .
$$

For any $p \in C_{+}(\bar{\Omega})$, denote

$$
1<p^{-}:=\inf _{x \in \bar{\Omega}} p(x) \leq p(x) \leq p^{+}:=\sup _{x \in \bar{\Omega}} p(x)<\infty .
$$

Let $p \in C_{+}(\bar{\Omega})$. Define the generalized Lebesgue space by

$$
L^{p(x)}(\Omega)=\left\{\left.u\left|u \in S(\Omega), \int_{\Omega}\right| u(x)\right|^{p(x)} \mathrm{d} x<\infty\right\},
$$

then $L^{p(x)}(\Omega)$ endowed with the norm

$$
|u|_{p(x)}=\inf \left\{\beta>0: \int_{\Omega}\left|\frac{u(x)}{\beta}\right|^{p(x)} \mathrm{d} x \leq 1\right\},
$$

becomes a Banach space.

Let $a \in S(\Omega)$, and $a(x)>0$ for a.e. $x \in \Omega$. Define the weighted variable exponent Lebesgue space $L_{a}^{p(x)}(\Omega)$ by

$$
L_{a}^{p(x)}(\Omega)=\left\{\left.u\left|u \in S(\Omega), \int_{\Omega} a(x)\right| u(x)\right|^{p(x)} \mathrm{d} x<\infty\right\},
$$

with the norm

$$
|u|_{p(x)}=\inf \left\{\beta>0: \int_{\Omega} a(x)\left|\frac{u(x)}{\beta}\right|^{p(x)} \mathrm{d} x \leq 1\right\} .
$$


From now on, we suppose that $a \in L^{\infty}(\Omega)$ and $\operatorname{essinf}_{x \in \Omega} a(x)=a_{0}>0$. Then obviously $L_{a}^{p(x)}(\Omega)$ is a Banach space (see [5] for details).

The variable exponent Sobolev space $W^{1, p(x)}(\Omega)$ is defined by

$$
W^{1, p(x)}(\Omega)=\left\{u \in L^{p(x)}(\Omega):|\nabla u| \in L^{p(x)}(\Omega)\right\}
$$

with the norm

$$
\|u\|=|u|_{p(x)}+|\nabla u|_{p(x)} .
$$

Next, the weighted-variable-exponent Sobolev space $W_{a}^{1, p(x)}(\Omega)$ is defined by

$$
W_{a}^{1, p(x)}(\Omega)=\left\{u \in L_{a}^{p(x)}(\Omega):|\nabla u| \in L_{a}^{p(x)}(\Omega)\right\}
$$

with the norm

$\|u\|_{a}=\inf \left\{\beta>0: \int_{\Omega}\left(\left|\frac{\nabla u(x)}{\beta}\right|^{p(x)}+a(x)\left|\frac{u(x)}{\beta}\right|^{p(x)}\right) \mathrm{d} x \leq 1\right\}, \forall u \in W_{a}^{1, p(x)}(\Omega)$.

Then the norms $\|\cdot\|_{a}$ and $\|\cdot\|$ in $W_{a}^{1, p(x)}(\Omega)$ are equivalent. If $1<p^{-} \leq p^{+}<$ $\infty$, then the space $W_{a}^{1, p(x)}(\Omega)$ is a separable and reflexive Banach space.

We set $\rho(u)=\int_{\Omega}\left(|\nabla u|^{p(x)}+a(x)|u|^{p(x)}\right) \mathrm{d} x$.

Proposition 1 ([7], Proposition 2.5). For all $u \in W_{a}^{1, p(x)}(\Omega)$, we have

(i) $\|u\|_{a} \leq 1 \Rightarrow\|u\|_{a}^{p^{+}} \leq \rho(u) \leq\|u\|_{a}^{p^{-}}$,

(ii) $\|u\|_{a} \geq 1 \Rightarrow\|u\|_{a}^{p^{-}} \leq \rho(u) \leq\|u\|_{a}^{p^{+}}$.

Remark 1. If $N<p^{-} \leq p(x)$ for any $x \in \Omega$, by Theorem 2.2. in [9] and the equivalence of the norms $\|\cdot\|_{a}$ and $\|\cdot\|$, we deduce that $W_{a}^{1, p(x)}(\Omega) \hookrightarrow$ $W_{a}^{1, p_{-}}(\Omega)$. Since $N<p^{-}$, it follows that $W_{a}^{1, p(x)}(\Omega) \hookrightarrow W_{a}^{1, p_{-}}(\Omega) \hookrightarrow \hookrightarrow C(\bar{\Omega})$. Defining the norm

$$
\|u\|_{\infty}=\sup _{x \in \bar{\Omega}}|u(x)|,
$$

then there exists a constant $k>0$ such that

$$
\|u\|_{\infty} \leq k\|u\|_{a}, \quad \forall u \in W_{a}^{1, p(x)}(\Omega) .
$$

To prove the existence of at least three weak solutions for each of the given problem $(\mathcal{P})$, we will use the following result proved in [15] that, on the basis of [2], can be equivalently stated as follows 
Theorem 1. Let $X$ be a separable and reflexive real Banach space; $\Phi: X \rightarrow$ $\mathbb{R}$ a continuously Gâteaux differentiable and sequentially weakly lower semicontinuous functional whose Gâteaux derivative admits a continuous inverse on $X^{*}, \Psi: X \rightarrow \mathbb{R}$ a continuously Gâteaux differentiable functional whose Gâteaux derivative is compact. Assume that

(i) $\lim _{\|u\| \rightarrow \infty} \Phi(u)+\lambda \Psi(u)=\infty$ for all $\lambda>0$;

and there are $r \in \mathbb{R}$ and $u_{0}, u_{1} \in X$ such that

(ii) $\Phi\left(u_{0}\right)<r<\Phi\left(u_{1}\right)$;

(iii) $\inf _{u \in \Phi^{-1}([-\infty, r])} \Psi(u)>\frac{\left(\Phi\left(u_{1}\right)-r\right) \Psi\left(u_{0}\right)+\left(r-\Phi\left(u_{0}\right)\right) \Psi\left(u_{1}\right)}{\Phi\left(u_{1}\right)-\Phi\left(u_{0}\right)}$

Then there exist an open interval $\Lambda \in(0, \infty)$ and a positive real number $q$ such that for each $\lambda \in \Lambda$ and every continuously Gatteaux differentiable functional $J: X \rightarrow \mathbb{R}$ with compact derivative, there exists $\sigma>0$ such that for each $\mu \in[0, \sigma]$, the equation

$$
\Phi^{\prime}(u)+\lambda \Psi^{\prime}(u)+\mu J^{\prime}(u)=0
$$

has at least three solutions in $X$ whose norms are less than $q$.

\section{The main result and proof of the theorem}

In this part, we will prove that for problem $(\mathcal{P})$ there also exist three weak solutions for the general case.

Definition 1. We say $u \in W_{a}^{1, p(x)}$ is a weak solution of problem $(\mathcal{P})$ if

$$
\begin{aligned}
\int_{\Omega}\left(|\nabla u|^{p(x)-2} \nabla u \nabla v+a(x)|u|^{p(x)-2} u\right) \mathrm{d} x-\lambda \int_{\Omega} & f(x, u) v \mathrm{~d} x \\
& -\mu \int_{\Omega} g(x, u) v \mathrm{~d} x=0
\end{aligned}
$$

for any $v \in W_{a}^{1, p(x)}$

Theorem 2. Assume that $p^{-}>N$ and $f(x, u)$ satisfies (f1), (f2). Then there exist an open interval $\Lambda \in(0, \infty)$ and a positive real number $q>0$ such that each $\lambda \in \Lambda$ and every function $g: \Omega \times \mathbb{R} \rightarrow \mathbb{R}$ which satisfying(f1), there exists $\delta>0$ such that for each $\mu \in[0, \delta]$ problem $(\mathcal{P})$ has at least three solutions whose norms are less than $q$. 
Proof. Let $X$ denote the weighted variable exponent Lebesgue space $W_{a}^{1, p(x)}(\Omega)$. Define

$$
F(x, t)=\int_{0}^{t} f(x, s) \mathrm{d} s \text { and } G(x, t)=\int_{0}^{t} g(x, s) \mathrm{d} s .
$$

In order to use Theorem 1, we define the functions $\Phi, \Psi, J: X \rightarrow \mathbb{R}$ by

$$
\begin{aligned}
\Phi(u) & =\int_{\Omega} \frac{1}{p(x)}\left(|\nabla u|^{p(x)}+a(x) u^{p(x)}\right) \mathrm{d} x \\
\Psi(u) & =-\int_{\Omega} F(x, u) \mathrm{d} x \\
J(u) & =-\int_{\Omega} G(x, u) \mathrm{d} x
\end{aligned}
$$

Arguments similar to those used in the proof of Proposition 3.1 in [14], we know $\Phi, \Psi, J \in C^{1}(X, \mathbb{R})$ with the derivatives given by

$$
\begin{aligned}
\left\langle\Phi^{\prime}(u), v\right\rangle & =\int_{\Omega}\left(|\nabla u|^{p(x)-2} \nabla u \nabla v+a(x) u^{p(x)-2} u v\right) \mathrm{d} x \\
\left\langle\Psi^{\prime}(u), v\right\rangle & =-\int_{\Omega} f(x, u) v \mathrm{~d} x \\
\left\langle J^{\prime}(u), v\right\rangle & =-\int_{\Omega} g(x, u) v \mathrm{~d} x
\end{aligned}
$$

for any $u, v \in X$. Thus, there exists $\lambda, \mu>0$ such that $u$ is a critical point of the operator $\Phi(u)+\lambda \Psi(u)+\mu J(u)$, that is $\Phi^{\prime}(u)+\lambda \Psi^{\prime}(u)+\mu J^{\prime}(u)=0$. For proving our result, it is enough to verify that $\Phi, \Psi$ and $J$ satisfy the hypotheses of Theorem 1.

It is obvious that $\left(\Phi^{\prime}\right)^{-1}: X^{*} \rightarrow X$ exists and continuous, because $\Phi^{\prime}$ : $X \rightarrow X^{*}$ is a homeomorphism by Lemma 2.2 in [12]. Moreover, $\Psi^{\prime}, J^{\prime}: X \rightarrow$ $X^{*}$ are completely continuous because of the assumption (f1) and [10], which imply $\Psi^{\prime}$ and $J^{\prime}$ are compact.

Next, we will verify that condition(i) of Theorem 1 is fulfilled. In fact, by Proposition 1, we have

$\Phi(u) \geq \frac{1}{p^{+}} \int_{\Omega}\left(|\nabla u|^{p(x)}+a(x)|u|^{p(x)}\right) \mathrm{d} x=\frac{1}{p^{+}} \rho(u) \geq \frac{1}{p^{+}}\|u\|_{a}^{p^{-}}, u \in X,\|u\|_{a}>1$.

On the other hand, due to the assumption (f1), we have

$$
\Psi(u)=-\int_{\Omega} F(x, u) \mathrm{d} x=\int_{\Omega}-F(x, u) \mathrm{d} x
$$


and

$$
|F(x, t)| \leq c_{1}|t|+c_{2} \frac{1}{\alpha(x)}|t|^{\alpha(x)}
$$

Therefore,

$$
\begin{aligned}
\Psi(u) & \geq-c_{1} \int_{\Omega}|u| \mathrm{d} x-c_{2} \int_{\Omega} \frac{1}{\alpha(x)}|u|^{\alpha(x)} \mathrm{d} x \\
& \geq-c_{3}\|u\|_{a}-\frac{c_{2}}{\alpha^{+}} \int_{\Omega}\left(|u|^{\alpha^{+}}+|u|^{\alpha^{-}}\right) \mathrm{d} x \\
& =-c_{3}\|u\|_{a}-c_{4}\left(|u|_{\alpha^{+}}^{\alpha^{+}}+|u|_{\alpha^{-}}^{\alpha^{-}}\right)
\end{aligned}
$$

Using Remark 1, we know that $X$ is continuously embedded in $L^{\alpha^{+}}$and $L^{\alpha^{-}}$. Furthermore, we can find two positive constants $d_{1}, d_{2}>0$ such that

$$
|u|_{\alpha^{+}} \leq d_{1}\|u\|_{a} \text { and }|u|_{\alpha^{-}} \leq d_{2}\|u\|_{a} \quad \forall u \in X
$$

Moreover

$$
\Psi(u) \geq-c_{3}\|u\|_{a}-c_{4} d_{1}\|u\|_{a}^{\alpha^{+}}-c_{4} d_{2}\|u\|_{a}^{\alpha^{-}}
$$

It follows that

$$
\Phi(u)+\lambda \Psi(u) \geq\left(\frac{1}{p^{+}}-\lambda c_{3}\right)\|u\|_{a}^{p^{-}}-\lambda c_{4}\left(d_{1}\|u\|_{a}^{\alpha^{+}}+d_{2}\|u\|_{a}^{\alpha^{-}}\right), \forall u \in X
$$

Since $1<\alpha^{+}<p^{-}$, then $\lim _{\|u\|_{a} \rightarrow \infty} \Phi(u)+\lambda \Psi(u)=\infty$ and (i) is verified.

In the following, we will verify the conditions (ii) and (iii) in Theorem 1. By $F_{t}^{\prime}(x, t)=f(x, t)$ and assumption (f2), it follows that $F(x, t)$ is increasing for $t \in\left(t_{0}, \infty\right)$ and decreasing for $t \in\left(0, t_{0}\right)$, uniformly with respect to $x$. Obviously, $F(x, 0)=0$. $F(x, t) \rightarrow \infty$ when $t \rightarrow \infty$, because of assumption (f2). Then there exists a real number $\delta>t_{0}$ such that

$$
F(x, t) \geq 0=F(x, 0) \geq F(x, \tau), \quad \forall x \in X, t>\delta, \tau \in\left(0, t_{0}\right) .
$$

Let $a, b$ be two real numbers such that $0<a<\min \left\{t_{0}, k\right\}$ with $k$ given in Remark 1 and $b>\delta$ satisfies

$$
b^{p^{-}}\|a\|_{L^{1}(\Omega)}>1
$$

and

$$
b^{p^{+}}\|a\|_{L^{1}(\Omega)}>1 \text {. }
$$

Let $b>1$. When $t \in[0, a]$, we have $F(x, t) \leq F(x, 0)$, it follows that

$$
\int_{\Omega} \sup _{0 \leq t \leq a} F(x, t) \mathrm{d} x \leq \int_{\Omega} F(x, 0) \mathrm{d} x=0
$$


Furthermore, we can get $\int_{\Omega} F(x, b) \mathrm{d} x>0$ because of $b>\delta$.

Moreover,

$$
\frac{1}{k^{p^{+}}} \frac{a^{p^{+}}}{b^{p^{-}}} \int_{\Omega} F(x, b) \mathrm{d} x>0 .
$$

The above two inequalities imply

$$
\int_{\Omega} \sup _{0 \leq t \leq a} F(x, t) \mathrm{d} x \leq 0<\frac{1}{k^{p^{+}}} \frac{a^{p^{+}}}{b^{p^{-}}} \int_{\Omega} F(x, b) \mathrm{d} x .
$$

Consider $u_{0}, u_{1} \in X$ with $u_{0}(x)=0$ and $u_{1}(x)=b$ for any $x \in \Omega$. We define $r=\frac{1}{p^{+}}\left(\frac{a}{k}\right)^{p^{+}}$. Clearly, $r \in(0,1)$. A simple computation implies

$$
\Phi\left(u_{0}\right)=\Psi\left(u_{0}\right)=0
$$

and

$$
\begin{gathered}
\Phi\left(u_{1}\right)=\int_{\Omega} \frac{1}{p(x)} a(x) b^{p(x)} \mathrm{d} x \geq \frac{1}{p^{+}} b^{p^{-}}\|a\|_{L^{1}(\Omega)}>\frac{1}{p^{+}}>\frac{1}{p^{+}}\left(\frac{a}{k}\right)^{p^{+}} \\
\Psi\left(u_{1}\right)=-\int_{\Omega} F\left(x, u_{1}(x)\right) \mathrm{d} x=-\int_{\Omega} F(x, b) \mathrm{d} x .
\end{gathered}
$$

Similarly for $b<1$, by help of Proposition 1, we get the desired result.

Thus, we obtain

$$
\Phi\left(u_{0}\right)<r<\Phi\left(u_{1}\right)
$$

and (ii) in Theorem 1 is verified.

On the other hand, we have

$$
-\frac{\left(\Phi\left(u_{1}\right)-r\right) \Psi\left(u_{0}\right)+\left(r-\Phi\left(u_{0}\right)\right) \Psi\left(u_{1}\right)}{\Phi\left(u_{1}\right)-\Phi\left(u_{0}\right)}=-r \frac{\Psi\left(u_{1}\right)}{\Phi\left(u_{1}\right)}=r \frac{\int_{\Omega} F(x, b) \mathrm{d} x}{\int_{\Omega} \frac{1}{p(x)} a(x) b^{p(x)} \mathrm{d} x}>0 .
$$

Next, we consider the case $u \in X$ with $\Phi(u) \leq r<1$. Since $\frac{1}{p(x)} \rho(u) \leq \Phi(u) \leq$ $r$, we obtain $\rho(u) \leq p^{+} r=\left(\frac{a}{k}\right)^{p^{+}}<1$, it follows that $\|u\|_{a}<1$. Furthermore, it is clear that

$$
\frac{1}{p^{+}}\|u\|_{a}^{p^{+}} \leq \frac{1}{p^{+}} \rho(u) \leq \Phi(u) \leq r .
$$

Thus, using Remark 1, we have

$$
|u(x)| \leq k\|u\|_{a} \leq k\left(p^{+} r\right)^{\frac{1}{p^{+}}}=a \quad \forall x \in \Omega, u \in X, \Phi(u) \leq r .
$$

The above inequality shows that

$$
\inf _{u \in \Phi^{-1}([-\infty, r])} \Psi(u)=\sup _{u \in \Phi^{-1}([-\infty, r])}-\Psi(u) \leq \int_{\Omega} \sup _{0 \leq t \leq a} F(x, t) \mathrm{d} x \leq 0 .
$$


It follows that

$$
\inf _{u \in \Phi^{-1}([-\infty, r])} \Psi(u)<r \frac{\int_{\Omega} F(x, b) \mathrm{d} x}{\int_{\Omega} \frac{1}{p(x)} a(x) b^{p(x)} \mathrm{d} x} .
$$

That is

$$
\inf _{u \in \Phi^{-1}([-\infty, r])} \Psi(u)>\frac{\left(\Phi\left(u_{1}\right)-r\right) \Psi\left(u_{0}\right)+\left(r-\Phi\left(u_{0}\right)\right) \Psi\left(u_{1}\right)}{\Phi\left(u_{1}\right)-\Phi\left(u_{0}\right)}
$$

which means that condition (iii) in Theorem 1 is verified. Then the proof of Theorem 2 is achieved.

Remark 2. Applying ([3], Theorem2.1) in the proof of Theorem 2, an upper bound of the interval of parameters $\lambda$ for which $(\mathcal{P})$ has at least three weak solutions is obtained when $\mu=0$. To be precise, in the conclusion of Theorem 2 one has

$$
\Lambda \subseteq] 0, h \frac{\int_{\Omega} \frac{1}{p(x)} a(x) b^{p(x)} \mathrm{d} x}{\int_{\Omega} F(x, b) \mathrm{d} x}[
$$

for each $h>1$ and $b$ as in the proof of Theorem 2.

\section{Acknowledgments}

The author would like to thank reviewers for clear valuable comments and suggestions. The first and the third author was supported by the Fundamental Research Funds for the Central Universities (No. XDJK2013D007), Scientific Research Fund of SUSE (No. 2011KY03) and Scientific Research Fund of SiChuan Provincial Education Department (No. 12ZB081).

\section{References}

[1] G. Anello and G. Cordaro. Existence of solutions of the Neumann problem for a class of equations involving the $p$-Laplacian via a variational principle of Ricceri. Arch. Math. (Basel), 79(4):274-287, 2002.

[2] G. Bonanno. A minimax inequality and its applications to ordinary differential equations. J. Math. Anal. Appl., 270(1):210-229, 2002.

[3] G. Bonanno. Some remarks on a three critical points theorem. Nonlinear Anal., 54(4):651-665, 2003.

[4] G. Bonanno and P. Candito. Three solutions to a Neumann problem for elliptic equations involving the $p$-Laplacian. Arch. Math. (Basel), 80(4):424-429, 2003. 
[5] D. Cruz-Uribe, L. Diening, and P. Hästö. The maximal operator on weighted variable lebesgue spaces. Frac. Calc. Appl. Anal., 14:361-374, 2011.

[6] G. Dai. Three solutions for a Neumann-type differential inclusion problem involving the $p(x)$-Laplacian. Nonlinear Anal., 70(10):3755-3760, 2009.

[7] X. Fan. Solutions for $p(x)$-Laplacian Dirichlet problems with singular coefficients. J. Math. Anal. Appl., 312(2):464-477, 2005.

[8] X. Fan, J. Shen, and D. Zhao. Sobolev embedding theorems for spaces $W^{k, p(x)}(\Omega)$. J. Math. Anal. Appl., 262(2):749-760, 2001.

[9] X. Fan and D. Zhao. On the spaces $L^{p(x)}(\Omega)$ and $W^{m, p(x)}(\Omega)$. J. Math. Anal. Appl., 263(2):424-446, 2001.

[10] O. Kováčik and J. Rákosník. On spaces $L^{p(x)}$ and $W^{k, p(x)}$. Czechoslovak Math. J., 41(116)(4):592-618, 1991.

[11] Q. Liu. Existence of three solutions for $p(x)$-Laplacian equations. Nonlinear Anal., 68(7):2119-2127, 2008.

[12] R. Mashiyev. Three Solutions to a Neumann Problem for Elliptic Equations with Variable Exponent. Arab. J. Sci. Eng., 36:1559-1567, 2011.

[13] M. Mihăilescu. Existence and multiplicity of solutions for a Neumann problem involving the $p(x)$-Laplace operator. Nonlinear Anal., 67(5):1419-1425, 2007.

[14] M. Mihăilescu and V. Rădulescu. A multiplicity result for a nonlinear degenerate problem arising in the theory of electrorheological fluids. Proc. R. Soc. Lond. Ser. A Math. Phys. Eng. Sci., 462(2073):2625-2641, 2006.

[15] B. Ricceri. A three critical points theorem revisited. Nonlinear Anal., 70(9):3084-3089, 2009.

[16] M. Růžička. Electrorheological fluids: modeling and mathematical theory, volume 1748 of Lecture Notes in Mathematics. Springer-Verlag, Berlin, 2000 .

[17] S. Samko. Denseness of $C_{0}^{\infty}\left(\mathbf{R}^{N}\right)$ in the generalized Sobolev spaces $W^{M, P(X)}\left(\mathbf{R}^{N}\right)$. In Direct and inverse problems of mathematical physics (Newark, DE, 1997), volume 5 of Int. Soc. Anal. Appl. Comput., pages 333-342. Kluwer Acad. Publ., Dordrecht, 2000. 
Wen-Wu PAN,

Department of Science,

Sichuan University of Science and Engineering,

Zigong 643000, P. R. China.

Email: 23973445@qq.com

Ghasem Alizadeh AFROUZI,

Department of Mathematics, Faculty of Mathematical sciences,

University of Mazandaran,

47416-1467 Babolsar, Iran.

Email: afrouzi@umz.ac.ir

Lin LI,

School of Mathematics and Statistics,

Southwest University,

Chongqing 400715, P. R. China.

Email: lilin420@gmail.com 